KYUNGPOOK Math. J. 52(2012), 375-381

http://dx.doi.org/10.5666/KMJ.2012.52.4.375

\title{
On Ordered Ternary Semigroups
}

\author{
VANITA Rohit DADDI \\ Department of Mathematics, D. Y. Patil College of Engineering and Technology, \\ Kolhapur, India \\ e-mail: vanita_daddi@rediffmail.com \\ YASHAShree ShIVAJIRAO PAWAR* \\ Department of Mathematics, SKN Sinhgad College of Engineering, Pandharpur, \\ Korti-413304, India \\ e-mail : pawar_y_s@yahoo.com
}

ABstract. We introduce the concepts of ordered quasi-ideals, ordered bi-ideals in an ordered ternary semigroup and study their properties. Also regular ordered ternary semigroup is defined and several ideal-theoretical characterizations of the regular ordered ternary semigroups are furnished.

\section{Introduction}

The literature of a ternary algebraic system was introduced by D. H. Lehmer [3] in 1932. He investigated certain ternary algebraic systems called triplexes which turn out to be ternary groups. The notion of ternary semigroup was known to S.Banach. He showed by an example that ternary semigroup does not necessarily reduce to an ordinary semigroup. In [6] M. L. Santiago developed the theory of ternary semigroups. He focused his attention mainly to the study of regular ternary semigroups, bi-ideals and ideals in ternary semigroups. The semigroup $\mathbb{Z}$ of all integers under multiplication which plays a vital role in the literature of semigroup. The subset $\mathbb{Z}^{+}$of all positive integers of $\mathbb{Z}$ is a semigroup under multiplication. Now if we consider the subset $\mathbb{Z}^{-}$of all negative integers of $\mathbb{Z}$, then it is not a semigroup under multiplication. Taking these facts in mind D. H. Lehmer [3] introduced the notion of ternary semigroup. $\mathbb{Z}^{-}$is a natural example of a ternary semigroup under the ternary multiplication. N. Kehayopulu in [5] developed the theory of po-semigroups. He mainly studied regular po-semigroups, ideals and bi-ideals in po-semigroups. In 1999, Sang Keun Lee and Seong Gon Kang [4] gave charac-

* Corresponding Author.

Received June 3, 2009; accepted July 13, 2012.

2010 Mathematics Subject Classification: 20N99, 06F99.

Key words and phrases: ordered semigroup, ordered ternary semigroup, ordered left ideal, ordered right ideal, ordered lateral ideal, ordered quasi-ideal, ordered bi-ideal, regular ordered ternary semigroup. 
terizations of the regular po-semigroups in terms of some special types of ideals. Aiyared Iampan [1] introduced an ordered ternary semigroup and characterized the minimality and maximality of ordered lateral ideals in ordered ternary semigroups.

In this paper an attempt is made to introduce the notion of ordered quasi-ideal and ordered bi-ideal in an ordered ternary semigroup as a generalization of the notion of ordered left, right, lateral ideals defined by Aiyared Iampan in an ordered ternary semigroup [1]. Also we define a regular ordered ternary semigroup. Our main purpose is to characterize regular ordered ternary semigroups.

\section{Preliminaries}

Lehmer [3] defined a ternary semigroup as follows.

Definition 1.1. A non-empty set $T$ together with a ternary operation [ ] defined on $T$ is called a ternary semigroup if [ ] satisfies the following associative law:

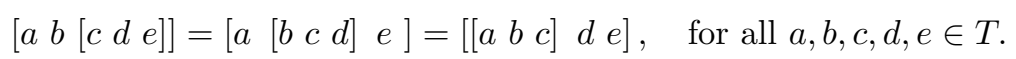

Every ordinary binary semigroup is a ternary semigroup under the same multiplication but not conversely. For this consider the following example.

Let $T=\{\ldots \ldots,-2 \mathrm{i},-\mathrm{i}, 0, \mathrm{i}, 2 \mathrm{i}, \ldots \ldots\} . T$ is a ternary semigroup under the multiplication over complex number. Note that this ternary semigroup does not reduce to an ordinary semigroup under the same multiplication.

By $[A B C]$, we mean the set $\left\{\left[\begin{array}{lll}a & b & c\end{array}\right] / a \in A, b \in B, c \in C\right\}$ for non-empty subsets $A, B, C$ of $T$. If $A=\{a\}$, then we write $[\{a\} B C]$ as $[a B C]$ and similarly if $B=\{b\}$ or $C=\{c\}$, we write $[A b C]$ and $[A B c]$ respectively.

Definition 1.2. A non-empty subset $S$ of a ternary semigroup $T$ is called a ternary subsemigroup of $T$, if $[S S S] \subseteq S$.

Definition 1.3. A ternary semigroup $T$ is called an ordered ternary semigroup if there exits a partially ordered relation $\leq$ such that for $a, b \in T$ if $a \leq b$ implies $[a x y] \leq[b x y],[x a y] \leq[x b y],[x y a] \leq[x y b]$, for all $x, y \in T$.

Example 1.4. The set $\mathbb{Z}^{-}$of all negative integers is an ordered ternary semigroup with respect to triple multiplication and a partial ordering relation $\leq$.

Now onwards $T=<T,[], \leq>$ denotes an ordered ternary semigroup. For $H \subseteq S$ we denote $(H]$ the subset of $T$ defined by

$$
(H]=\{t \in T / t \leq h, \quad \text { for some } h \in H\} .
$$

Definition 1.5. A non-empty subset I of $T$ is called an ordered left ( respectively an ordered right, an ordered lateral) ideal of $T$ if

(1) $[T T \mathrm{I}] \subseteq \mathrm{I}\left(\operatorname{respectively}[\mathrm{I} T T] \subseteq \mathrm{I},\left[\begin{array}{ll}T & \mathrm{I}\end{array}\right] \cup\left[\begin{array}{ll}T T & \mathrm{I}\end{array}\right] \subseteq \mathrm{I}\right)$,

(2) For $a \in \mathrm{I}, b \in T$ such that $b \leq a$ implies $b \in I$, that is $(I]=\mathrm{I}$. 
Definition 1.6. (a) A non-empty subset $I$ of $T$ is called a two-sided ordered ideal of $T$ if $I$ is both an ordered left and an ordered right ideal of $T$.

(b) A non-empty subset $I$ of $T$ is called an ordered ideal if $I$ is an ordered left, an ordered right and an ordered lateral ideal of $T$.

We denote by $L(A), R(A), M(A)$ and $I(A)$ the ordered left ideal, ordered right ideal, ordered lateral ideal and ordered ideal of $T$, respectively generated by a non-empty subset $A$ of $T$, that is the least, with respect to the inclusion relation, ordered left ideal, ordered right ideal, ordered lateral ideal and ordered ideal of $T$, respectively containing $A$. As usual $L(A)$ (respectively $R(A), M(A)$ ) coinsides with the intersection of all ordered left (respectively ordered right, ordered lateral) ideals of $T$ containing $A, \mathrm{I}(A)$ is the intersection of all ordered ideals of $T$ containing $A$.

Following properties are observed.

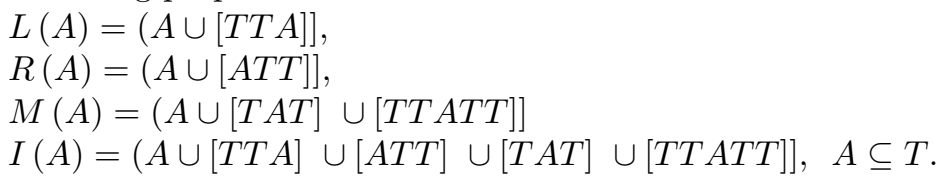

For $\{a\}$, we write $L(a), R(a), M(a)$ and $I(a)$ simply for $L(\{a\}), R(\{a\}), M(\{a\})$ and $I(\{a\})$ respectively,and we call them the ordered principal left ideal,the ordered principal right ideal, the ordered principal lateral ideal and principal ordered ideal of $T$ respectively generated by $a \in T$. Further we have,

$$
\begin{aligned}
L(a) & =\{t \in T / t \leq a \text { or } t \leq[x y a], \text { for some } x, y \in T\} \\
& =(a \cup[T T a]] \\
& =(a] \cup([T T a]] . \\
R(a) & =\{t \in T / t \leq a \text { or } t \leq[a x y], \text { for some } x, y \in T\} \\
& =(a \cup[a T T]] \\
& =(a] \cup([a T T]] . \\
M(a) & =\{t \in T / t \leq a \text { or } t \leq[x a y] \text { or } t \leq[x y a p q] \text { for some } x, y, p, q \in T\} \\
& =(a \cup[T a T] \cup[T T a T T]] \\
& =(a] \cup([T a T]] \cup([T T a T T]] .
\end{aligned}
$$

$I(a)=\{t \in T / t \leq a$ or $t \leq[x y a]$, or $t \leq[y x a]$ or $t \leq[x a y]$ or $t \leq[x y a p q]$, for some $x, y, p, q \in T\}=(a] \cup([T T a]] \cup([a T T]] \cup([T a T]] \cup([T T a T T]]$.

Definition 1.7. An ordered ternary semigroup $T$ is called an ordered left(right or lateral) simple if it has no proper ordered left (right or lateral) ideal of $T$.

Theorem 1.8. Let $T$ be an ordered ternary semigroup, then the following hold

1) $A \subseteq(A]$, for all $A \subseteq T$.

2) If $A \subseteq B \subseteq T$, then $(A] \subseteq(B]$.

3) $((A]]=(A]$, for all $A \subseteq T$.

4) $(A](B](C] \subseteq([A B C]]$, for all $A, B, C \subseteq T$. 
5) For any $a \in T,([T T a]],([T a T]] \cup([T T a T T]]$ and $([a T T]]$ are ordered left, ordered lateral and ordered right ideal of $T$ respectively.

6) For any $a \in T$, the set ([TTaTT]] is an ordered ideal of $T$.

\section{Ordered quasi-ideals and ordered bi-ideals}

Definition 2.1. A non-empty subset $Q$ of $T$ is called an ordered quasi-ideal of $T$ if

(i) $[Q T T] \cap[T Q T] \cap[T T Q] \subseteq \mathrm{Q}$,

(ii) $[Q T T] \cap[T T Q T T] \cap[T T Q] \subseteq \mathrm{Q}$,

(iii) For $a \in Q$ and $b \in T$ such that $b \leq a$ implies $b \in Q$. i.e. $(Q]=Q$.

For $a \in T$, an ordered quasi-ideal of $T$ generated by $a$ is denoted by $Q(a)$.

It is the smallest ordered quasi-ideal of $T$ containing $a$.

That is $Q(a)=(a \cup(\{[a T T] \cap([T a T] \cup[T T a T T]) \cap[T T a]\}]]$

Definition 2.2. A non-empty subset $B$ of $T$ is called an ordered bi-ideal of $T$ if

(i) $[B T B T B] \subseteq B$,

(ii) for $a \in B, b \in T$ such that $b \leq a$ implies $b \in B$ i.e. $(B]=B$.

For $a \in T$, an ordered bi-ideal of $T$ generated by $a$ is denoted by $B(a)$,

It is the smallest ordered bi-ideal of $T$ containing $a$.

That is $B(a)=(a \cup[a T a T a]]$

Definition 2.3. An ordered bi-ideal $B$ of $T$ is called sub-idempotent ordered biideal if $B^{3}=[B B B] \subseteq B$.

Remark 2.4. Every ordered ideal of $T$ is an ordered quasi-ideal of $T$, but every ordered quasi-ideal need not be an ordered ideal of $T$. We illustrate this in the following example.

Let $T=\{0, a, b, c, d\}$. Define the binary operation ( ) on $T$ as given in the following table.

\begin{tabular}{|l|l|l|l|l|l|}
\hline() & 0 & $a$ & $b$ & $c$ & $d$ \\
\hline 0 & 0 & 0 & 0 & 0 & 0 \\
\hline$a$ & 0 & $a$ & $b$ & 0 & 0 \\
\hline$b$ & 0 & 0 & 0 & $a$ & $b$ \\
\hline$c$ & 0 & $c$ & $d$ & 0 & 0 \\
\hline$d$ & 0 & 0 & 0 & $d$ & $d$ \\
\hline
\end{tabular}

Define the ternary operation [ ] on $T$ by, $[a b c]=(a(b c))=((a b) c)$, for all $a, b, c \in T$ and with the order $\leq:=\{(0,0),(a, a),(b, b),(c, c),(d, d),(0, a),(0, c)\}$.

The set $Q=\{0, b\}$ is an ordered quasi-ideal of $T$ which is not an ordered ideal of $T$.

We now state some elementary properties of ordered quasi-ideals and ordered bi-ideals without proof.

1. Intersection of any family of ordered quasi-ideals of $T$ is an ordered quasi-ideal of $T$, provided it is non-empty. 
2. If $S$ is an ordered ternary subsemigroup and $Q$ is an ordered quasi-ideal of $T$, then $Q \cap S$ is an ordered quasi-ideal of $S$.

3. If $A$ is an ordered ideal and $Q$ is an ordered quasi-ideal of $T$, then $A \cap Q$ is an ordered quasi-ideal of $T$.

4. Every ordered ideal of $T$ is an ordered bi-ideal of $T$.

5. Every ordered quasi-ideal of $T$ is an ordered bi-ideal of $T$.

6. Intersection of any family of ordered bi-ideals of $T$ is an ordered bi-ideal of $T$, provided it is non-empty.

7. If $S$ is an ordered ternary subsemigroup and $B$ is an ordered bi-ideal of $T$, then $B \cap S$ is an ordered bi-ideal of $S$.

Theorem 2.5. If $B$ is an ordered bi-ideal and $T_{1}, T_{2}$ are two ordered ternary subsemigroups of $T$, then $\left(\left[B T_{1} T_{2}\right]\right],\left(\left[T_{1} B T_{2}\right]\right]$ and $\left(\left[T_{1} T_{2} B\right]\right]$ are ordered bi-ideals of $T$.

Proof. $\left(\left[\left(\left[B T_{1} T_{2}\right]\right] T\left(\left[B T_{1} T_{2}\right]\right] T\left(\left[B T_{1} T_{2}\right]\right]\right]\right]$

$\subseteq\left(\left[\left[B T_{1} T_{2}\right] T\left[B T_{1} T_{2}\right] T\left[B T_{1} T_{2}\right]\right]\right]$

$\subseteq\left(\left[B\left[T_{1} T_{2} T\right] B\left[T_{1} T_{2} T\right]\left[B T_{1} T_{2}\right]\right]\right]$

$\subseteq\left(\left[[B T B T B] T_{1} T_{2}\right]\right]$

$\subseteq\left(\left[B T_{1} T_{2}\right]\right]$ (since $B$ is an ordered bi-ideal).

Let $a \in\left(\left[B T_{1} T_{2}\right]\right]$ and $x \in T$. Then $a \leq\left[b t_{1} t_{2}\right]$, for some $\left[b t_{1} t_{2}\right] \in\left[B T_{1} T_{2}\right]$ such that $x \leq a$, therefore $x \leq\left[b t_{1} t_{2}\right]$ and hence $x \in\left(\left[B T_{1} T_{2}\right]\right]$. Therefore $\left(\left[B T_{1} T_{2}\right]\right]$ is an ordered bi-ideal of $T$. Similarly we can prove that $\left(\left[T_{1} B T_{2}\right]\right]$ and $\left(\left[T_{1} T_{2} B\right]\right]$ are ordered bi-ideal of $T$.

Theorem 2.6. If $B_{1}, B_{2}$ and $B_{3}$ are ordered bi-ideals of $T$, then $\left(\left[B_{1} B_{2} B_{3}\right]\right]$ is an ordered bi-ideal of $T$.

Definition 2.7. An element $a \in T$ is called regular if there exists $x \in T$ such that $a \leq[a x a]$. If every element of $T$ is regular, then $T$ is called regular ordered ternary semigroup.

Theorem 2.8. Let $S$ be an ordered ternary subsemigroup of $T$. Then $S$ is regular if and only if $a \in([a S a]]$, for all $a \in S$.

Proof. Suppose $S$ is regular. Then for $a \in S$ there exists $x \in S$ such that $a \leq[a x a]$. Since $[a x a] \in[a S a], a \in([a S a]]$.

Conversely, let $a \in([a S a]]$ for $a \in S$. As $a \leq[a x a]$ for some $x \in S, a$ is regular. This shows that $S$ is regular.

Theorem 2.9. If $T$ is both ordered left and ordered right simple, then $T$ is regular.

Proof. Let $a \in T$. Then $([T T a]]$ and $([a T T]]$ are left and right ideals of $T$ respectively. But $T$ is left simple and right simple, therefore $([T T a]]=T$ and $([a T T]]=T$. 
We have $a \in([a T T]]=([a T([T T a]]]]=([a[T T T] a]] \subseteq([a T a]]$. Therefore $T$ is regular.

Theorem 2.10. If $T$ is regular, then an ordered bi-ideal is a sub-idempotent ordered bi-ideal of $T$ and conversely.

Proof. Let $B$ be an ordered bi-ideal of $T$. Then $[B T B T B] \subseteq B,([B T B T B]] \subseteq(B]=$ $B$. Since $T$ is regular,we have $B \subseteq([B T B]] \subseteq([B T([B T B]]]])([B T B T B]]$.

Thus $B^{3}=[B B B]$

$$
\begin{aligned}
& \subseteq[([B T B T B]]([\text { BTBTB B B }]]([B T B T B]]] \\
& \subseteq([B T B T B][B T B T B][B T B T B]] \\
& =([B[T B T] B[B T B T B] B[T B T] B]] \\
& \subseteq([B[T T T] B B B[T T T] B]] \\
& \subseteq([[B T B][B B T] B]] \\
& \subseteq([[B T B][T T T] B]] \\
& \subseteq([B T B T B]] \\
& \subseteq(B]=B .
\end{aligned}
$$

Hence $B$ is sub-idempotent ordered bi-ideal of $T$. As $B$ is sub-idempotent ordered bi-ideal of $T, B$ is an ordered bi-ideal of $T$.

Theorem 2.11. $T$ is regular if and only if for every ordered bi-ideal $B$, an ordered lateral ideal $M$ and an ordered left ideal $L$ of $T$, we have $B \cap M \cap L \subseteq([B M L]]$.

Proof. If $T$ is regular, then for any $a \in B \cap M \cap L$ there exists $x \in T$ such that $a \leq[$ axa $] \leq[[$ axa $]$ xa $] \leq[[$ axaxa $] x[$ axa $]] \leq[[$ axaxa $][$ xax $] a] \in[[B T B T B][T M T] L] \subseteq$ $[B M L]$ (since $B$ is an ordered bi-ideal, $M$ is an ordered lateral ideal and $L$ is an ordered left ideal of $T)$.

Hence $a \in([B M L]]$. Therefore $B \cap M \cap L \subseteq([B M L]]$.

Conversely suppose that $B \cap M \cap L \subseteq([B M L]]$, for every ordered bi-ideal $B$, every ordered lateral ideal $M$ and every ordered left ideal $L$ of $T$. Let $a \subseteq T$. Consider ordered bi-ideal $B(a)$, ordered lateral ideal $M(a)$ and ordered left ideal $L(a)$ of $T$ generated by $a$. Then we have, $a \subseteq B(a) \cap M(a) \cap L(a) \subseteq([B(a) M(a) L(a)]]$

$\subseteq((a \cup[a T a T a]] T(a \cup[T T a]]]$

$\subseteq([a T a] \cup[a T T T a] \cup[a T a T a T a] \cup[a T a T a T T T a]] \subseteq([a T a]]$.

Thus $T$ is regular.

As in Theorem 2.11, we have the following:

Theorem 2.12. For an ordered ternary semigroup $T$ the following statements are equivalent:

1. $T$ is regular.

2. $B \cap I \cap L \subseteq([B I L]]$ for an ordered bi-ideal $B$, an ordered ideal $I$ and an ordered left ideal $L$ of $T$.

3. $R \cap M \cap B=([R M B]]$ for an ordered bi-ideal $B$, an ordered lateral ideal $M$ and an ordered right ideal $R$ of $T$. 
4. $R \cap I \cap B=([R I B]]$ for an ordered bi-ideal $B$, an ordered ideal $I$ and an ordered right ideal $R$ of $T$.

5. $B \cap I \cap Q \subseteq([B I Q]]$ for an ordered bi-ideal $B$, an ordered ideal $I$ and an ordered quasi-ideal $Q$.

6. $R \cap M \cap L \subseteq([R M L]]$ for an ordered right ideal $R$, an ordered lateral ideal $M$ and an ordered left ideal $L$ of $T$.

\section{References}

[1] Iampan Aiyared, Characterizing the minimality and maximality of ordered lateral ideals in ordered ternary semigroups, J. Korean Math. Soc., 46(4)(2009), 775-784.

[2] V. N. Dixit and Sarita Diwan, A note on quasi and Bi-ideals in ternary semigroups, Int. J. Math. Sci., 18(1995), 501-508.

[3] D. H. Lehmer, A ternary analogue of abelian groups, Ams. J. Math., 59(1932), 329338.

[4] S. K. Lee and S. G. Kang, Characterization of Regular po-semigroups, Comm. Korean Math. Soc., 14(4)(1999), 687-691.

[5] N. Kehayopulu, J. S. Ponizovskii and M. Tsingelis, Bi-ideals in ordered semigroups and ordered groups, Jou. of Math. Sci., 112(4)(2002).

[6] M . L. Santigo, Regular ternary semigroups, Bull. Cal. Math. Soc., 82(1990), 67-71l.

[7] F. M. Sioson, Ideal Theory in ternary semigroups, Math. Japan., 10(1965), 63-84. 